\title{
FACTOR ANALYSIS AND ITS APPLICATION IN INNOVATION MANAGEMENT WITHIN MANUFACTURING ENTERPRISES IN ROMANIA
}

\author{
Lucia Michalkova $^{1, a, *}$, Katarina Frajtova Michalikova ${ }^{2, b}$ and Alina Tănase ${ }^{3, c}$ \\ ${ }^{1}$ Department of Economics, Faculty of Operation and Economics of Transport and Communications, \\ University of Zilina, Univerzitna 1, 01026 Zilina, Slovak Republic \\ ${ }^{2}$ Department of Economics, Faculty of Operation and Economics of Transport and Communications, \\ University of Zilina, Univerzitna 1, 01026 Zilina, Slovak Republic \\ ${ }^{3}$ The Management Doctoral School, The Bucharest University of Economic Studies, 6 Piata Romania, \\ $1^{\text {st }}$ district, Bucharest, 010374 Romania \\ alucia.michalkova@fpedas.uniza.sk, bfmichalikova@fpedas.uniza.sk, cemail4alina@yahoo.com \\ *Corresponding author
}

Cite as: MICHALKOVA, L., FRAJTOVA MICHALIKOVA, K., TĂNASE, A. (2019). Factor analysis and its application in innovation management within manufacturing enterprises in Romania. Ekonomickomanazerske spektrum, 13(2), 37-45.

Available at: dx.doi.org/10.26552/ems.2019.2.37-45

\begin{abstract}
Business management plays a vital role in building processes, structures, and the business environment to make the business innovative and to motivate individuals and teams to innovate. The competitive market, together with the pressure of international competition, emphasizes the importance of businesses successfully managing innovation-enhancing activities. However, innovation management is a complex theme, and its development involves several uncertainties and risks, such as failed ideas, the degree of human motivation, unexpected changes in market requirements, and more. Recently, foreign researchers, in particular, have paid great attention to identifying and exploiting the main incentives for enterprise-level innovation management, leading to many research articles that have adopted different approaches and identified a large number of innovation-related factors. To identify critical factors, factor analysis was used to summarize the data so that relationships and formulas could be easily interpreted and understood. It is commonly used to regroup variables into a limited set of clusters based on the shared variation. Therefore, it helps to isolate constructs and concepts. Knowing the key factors of innovation that can provide sustainable growth and competitiveness to a business is essential, but not least, the primary role of management should be to evaluate innovation investments. Within the current state of the problem, 17 critical factors of innovation were identified through factor analysis. The results have also been demonstrated on a model example of the dependence between $R \& D$ costs and significant innovation factors for Romanian enterprises.
\end{abstract}

Keywords: Innovation, innovation management, factor analysis, R\&D

JEL Classification: O14, O32, Q55

\section{Introduction}

Factor analysis originated in the early 20th century, when Charles Spearman was interested in human capabilities and the evolution of the two-factor theory, ultimately leading to increased work on the opinions and mathematical principles of factor analysis Thompson (1993). The 
method included simulated data where responses were already known for testing factor analysis. Factor analysis appears in various areas such as behavioural and social sciences, medicine, economics, and geography as a result of the technological advancement of computers. Large data sets, which consist of several variables, can be reduced by observing them within certain "groups" of variables or factors, respectively. Factor analysis compiles common variables into descriptive categories. Factor analysis is useful for studies that include several or hundreds of variables, questionnaire items, or a set of tests that can be reduced to a smaller set, get to the basic concept, and facilitate interpretation. It is easier to focus on some key factors rather than having to take into account too many quantities of variables that can be trivial. Therefore, factor analysis is useful for placing variables into meaningful categories.

The manager must successfully create a synergy between the innovator's abilities and the external characteristics of the environment Schewe (1991). Therefore, leaders or top management need to identify different factors for innovation at different levels and take appropriate measures at organizational and group levels to create a culture that fosters innovation at an individual level, which in turn facilitates innovation at the organizational level Walker et al. (2011). Besides, it is essential to identify individuals with appropriate expertise and delegate appropriate measures to them.

Every business that wants to be successful in the long term needs an innovation management system to avoid being uncontrolled. Innovation management creates structures and framework conditions so that innovation potential can be systematically identified, ideas designed, and then successfully implemented Schepers et al. (2016). The scale of innovation management also depends on the size of the company. Small and medium-sized enterprises usually do not have explicit innovation processes and tasks but are represented in existing structures or within the project. For managing innovation is necessary to identify the factors that ensure innovation at each level Kratzer et al. (2017).

Market research and comprehensive data analysis are needed to analyse and identify the right market segment, potential demand, and potential areas of competition, product development requirements, and all other aspects of the trading book. One of the most common tools used to deal with large amounts of data is factor analysis according to Kang and Lee (2017) and Coccia (2017). Factor analysis is a multivariate statistical method based on a review of the structure of interdependencies of variables based on the assumption that these dependencies are due to the action of a certain number of underlying non-measurable factors called common factors Goodwyn (2012). Factor analysis provides a diagnostic tool to assess whether the collected data is consistent with the theoretically expected pattern or structure of the target construct. Factor analysis explains the relationship between two observable variables and how another smaller set of unobservable variables influences them, Garcia-Granero et al. (2015). It is a technique applied to a set of monitored variables, which tries to find the essential factors (subsets of variables) from which the monitored variables created. By this method, it is possible to detect hidden lateral causes, which are also sources of data variability, Foroudi et al. (2016). For example, an individual's response to a university entrance test was influenced by essential variables such as intelligence, years spent at school, age, and emotional state on the day of the trial, the amount of test practice, etc. Variables follow the answers to the questions. Basic, influential variables are factors. Factor analysis performed on the correlation matrix of the variables of interest. The factor is the weighted average of the original variables, Kaasa (2016). It is necessary to find several elements from which to create the original correlation matrix. Usually, the goal of factor analysis is to support the interpretation of data, according to Dobni (2008). 


\section{Methodology}

The main aim of the research paper is to propose a list of key innovation factors and to create a model enabling to predict the impact of significant innovation factors on the costs of research and development for Romanian enterprises. An important tool is the factor analysis that we decided to use to clarify the goal, Cooper and Edgett (2010). Factor analysis is an interdependent technique in which all correlating variables are considered simultaneously. In a sense, each of the observed (original) variables is considered to be a dependent variable that is a function of some basic, latent, and hypothetical set of factors (dimensions), Klami et al. (2015). Conversely, each factor is a function of the variables initially observed. Factor analysis is often used to process questionnaire data in the case of capability measurements or features; it is necessary to make sure that the questions relate to the construction we want to measure.

We designed a questionnaire to measure different aspects of performance in innovation management. We created questions by analysing publications in peer-reviewed journals and got answers from 155 Romanian enterprises. Each issue was a statement, followed by a six-point Likert scale. Therefore, there is no reason to assume significant differences in distances between individual values. Likert scales are one of the most reliable ways to measure opinions, perceptions, and behaviour, in accordance with Abbas and Raja (2015).

We assume that $x_{T}=\left(x_{1}, x_{2}, \ldots, x_{p}\right) T$ is one object described by $\mathrm{p}$ observed variables, with the general model of factor analysis assuming that there exists $m$ in the background of the common factors $F_{1}, F_{2}, \ldots, F m$, less than $p$. Then the object can be written as a linear combination of common factors as follows, Kano and Harada (2000):

$$
\begin{gathered}
x_{1}=l_{11} F_{1}+l_{12} F_{2}+\cdots+l_{1 m} F_{m}+e_{1} \\
x_{2}=l_{21} F_{1}+l_{22} F_{2}+\cdots+l_{2 m} F_{m}+e_{2} \\
\cdots \\
x_{p}=l_{p 1} F_{1}+l_{p 2} F_{2}+\cdots+l_{p m} F_{m}+e_{p}
\end{gathered}
$$

where:

$x=\left(x_{1}, x_{2}, \ldots, x_{p}\right) \quad p$ dimensional, random vector,

$F_{1}, F_{2}, \ldots, F_{m} \quad$ common factors,

$l_{i k} \quad$ factor loadings,

$e_{1}, e_{2}, \ldots, e_{p} \quad$ specific factors (model errors).

$F_{1}, F_{2}, \ldots, F m$ referred to as common factors causing correlations between $p$ original variables. The mean of these factors is zero and the unit variance Neumann et al. (2017). In this model, we would also find error components $e_{1}, e_{2}, \ldots e_{p}$, which contributes to the dispersion of individual variables. The coefficients $l_{i k}$ are used to denote the factor loadings of the $i$-th variable on the $j$-th common factor $F j, i=1, \ldots, p, j=1, \ldots, m$. In other words, the factor loads lij, if the same measurement units are assumed, can be interpreted as a contribution of the $j$ factor of the $i$-th variable explained, Dobni (2008). Factor burdens, when certain solution conditions are met, represent covariations or correlations between original and new variables and we can rewrite the factor model in a matrix form as:

$$
X=F L^{T}+E
$$

where:

$\mathrm{X} \quad$ matrix dimension $n \times p$, 


$$
\begin{array}{ll}
\text { F matrix dimension } n \times m, \\
\mathrm{~L} \text { matrix of factor loads, } \\
\mathrm{E} \text { error matrix. }
\end{array}
$$

$X$ is understood as a data matrix of dimension $n x p, F$ represents a matrix of dimension $n$ $x \mathrm{~m}$, where the common factors $F_{1}, F_{2}, \ldots F_{m}$ are arranged in columns, $L$ shows a matrix of factor loadings of size $p \times m$ and $E$ is an error matrix with dimension $n \times p$ and columns are made up of specific factors $e_{1}, e_{2}, \ldots e_{p}$ Dobni (2008).

The essential factor theorem in the form of covariance matrix $S$ of input variables (i.e., columns of data matrix $X$ ), whose dimension is $p x p$, for orthogonal factor model looks like:

$$
S=L L^{T}+\Gamma^{2}
$$

where:

S covariance matrix $S$ of input variables,

$L L^{T} \quad$ covariance matrix of $\mathrm{FL}^{\mathrm{T}}$,

$\Gamma^{2} \quad$ covariance matrix of error factors.

Where $\mathrm{LL}^{\mathrm{T}}$ is the covariance matrix of the columns of the $\mathrm{FL}^{\mathrm{T}}$ matrix, where $\left(F L^{T}\right)^{T} *\left(F L^{T}\right)=L F^{T} F^{T}=L^{T}$, since the covariance matrix of common $\mathrm{F}^{\mathrm{T}} \mathrm{F}$ is a unit matrix because the factors are uncorrelated and have unit variance. The matrix $\Gamma 2$ is a covariance matrix of error factors, referred to uniqueness matrix, and is a diagonal matrix due to the assumption of uncorrelated errors.

Based on the solution of factor analysis, we can estimate factor scores. The factor score is represented by the values of the factors, respectively, of the non-measurable, latent variables. If the range of observations is $\mathrm{n}$, then the factor score solution is a matrix of factor scores. The factor score is not an estimate in the ordinary sense, because it is an estimate of non-measurable variables. Factor saturation estimates and specific variance estimates are used to estimate factor scores. Assessments can be based on both a non-rotated and a rotated solution. There are several methods to determine the factor score. The most commonly used are Regression Method and Bartlett Scores.

The necessary information for the creation of the database was obtained through a questionnaire designed by prof. Milé Terziovsky, $\mathrm{PhD}$. from the University of South Australia for a study entitled "Assessing the Innovative Capability of Models for Creating Innovative Enterprises" (Terziovsky, 2010). The author sent the questionnaire through Australia, and each question was tested and reviewed until the pilot version of the study was the final version of the survey with innovation factors. In recent years, innovation became the main stimulus for the economic growth of the economies of advanced industrialized nations of the world and given that change is a generator of new products, we have focused primarily on Romanian industrial manufacturing companies. The sample was, therefore, not random, it was a deliberate or purposeful sample. Companies had to meet the following conditions:

- they have been operating on the market for more than three years,

- business focused on industrial production,

- belongs to SMEs.

The original sample of Professor Terziovski's study also focuses on industrial production, specifically on manufacturing, services, computers, and construction. The author created a database of thousands of businesses, using a Dunn \& Bradstreet data file with a total of 20,000 companies defined by industry-standard classification by systematic random sampling. In our case, we created a database of 155 Romanian companies. 


\section{Results}

For processing factor analysis, the selection of input data and the assessment of the suitability of these data was necessary. Data were obtained through electronic querying. The total number of survey questions was 72 , and they were formulated so that respondents could choose an answer on a scale, which allowed the solutions transformed into numerical values necessary for processing factor analysis.

The use of factor analysis is conditional on the assumption that the input variables are correlated with each other to confirm the existence of common causes. Interdependencies and evaluation of input variables were verified by the Kaiser - Meyer - Olkin (KMO) criterion. The KMO criterion compares both single and partial correlation coefficients. Since the issue was dealt with in the SPSS program, we also performed the Bartlett test. The next important step is to determine the number of common factors. There are several ways to estimate this number, and the initial choice may not be final but may change during the calculations. One of these is the criterion of the percentage of explained variability that we investigated. In Table 1, we included $72.402 \%$ of the explained variability through 17 common factors.

Table 1: Total Variance Explained

\begin{tabular}{|c|c|c|c|c|c|c|c|c|c|}
\hline \multirow[b]{2}{*}{ Component } & \multicolumn{3}{|c|}{ Initial Eigenvalues } & \multicolumn{3}{|c|}{$\begin{array}{l}\text { Extraction Sums of Squared } \\
\text { Loadings }\end{array}$} & \multicolumn{3}{|c|}{$\begin{array}{l}\text { Rotation Sums of Squared } \\
\text { Loadings }\end{array}$} \\
\hline & Total & $\begin{array}{l}\text { Variance } \\
\%\end{array}$ & $\begin{array}{l}\text { Cumulative } \\
\%\end{array}$ & Total & $\begin{array}{l}\text { Variance } \\
\%\end{array}$ & $\begin{array}{l}\text { Cumulative } \\
\%\end{array}$ & Total & $\begin{array}{l}\text { Variance } \\
\%\end{array}$ & $\begin{array}{l}\text { Cumulative } \\
\%\end{array}$ \\
\hline 1 & 19.774 & 27.463 & 27.463 & 19.774 & 27.463 & 27.463 & 7.768 & 10.789 & 10.789 \\
\hline 2 & 5.188 & 7.206 & 34.669 & 5.188 & 7.206 & 34.669 & 6.047 & 8.399 & 19.188 \\
\hline 3 & 3.060 & 4.250 & 38.919 & 3.060 & 4.250 & 38.919 & 4.936 & 6.856 & 26.044 \\
\hline 4 & 2.898 & 4.025 & 42.944 & 2.898 & 4.025 & 42.944 & 4.798 & 6.664 & 32.707 \\
\hline 5 & 2.556 & 3.550 & 46.495 & 2.556 & 3.550 & 46.495 & 3.666 & 5.091 & 37.798 \\
\hline 6 & 2.324 & 3.228 & 49.722 & 2.324 & 3.228 & 49.722 & 3.613 & 5.018 & 42.816 \\
\hline 7 & 2.225 & 3.090 & 52.812 & 2.225 & 3.090 & 52.812 & 3.007 & 4.176 & 46.993 \\
\hline 8 & 1.804 & 2.506 & 55.318 & 1.804 & 2.506 & 55.318 & 2.718 & 3.775 & 50.768 \\
\hline 9 & 1.665 & 2.313 & 57.630 & 1.665 & 2.313 & 57.630 & 2.633 & 3.657 & 54.424 \\
\hline 10 & 1.543 & 2.143 & 59.774 & 1.543 & 2.143 & 59.774 & 1.800 & 2.501 & 56.925 \\
\hline 11 & 1.464 & 2.034 & 61.807 & 1.464 & 2.034 & 61.807 & 1.761 & 2.446 & 59.371 \\
\hline 12 & 1.426 & 1.980 & 63.788 & 1.426 & 1.980 & 63.788 & 1.734 & 2.408 & 61.779 \\
\hline 13 & 1.358 & 1.887 & 65.674 & 1.358 & 1.887 & 65.674 & 1.603 & 2.226 & 64.006 \\
\hline 14 & 1.280 & 1.778 & 67.452 & 1.280 & 1.778 & 67.452 & 1.580 & 2.195 & 66.200 \\
\hline 15 & 1.247 & 1.732 & 69.184 & 1.247 & 1.732 & 69.184 & 1.565 & 2.173 & 68.373 \\
\hline 16 & 1.218 & 1.692 & 70.875 & 1.218 & 1.692 & 70.875 & 1.551 & 2.154 & 70.527 \\
\hline 17 & 1.099 & 1.526 & 72.402 & 1.099 & 1.526 & 72.402 & 1.350 & 1.875 & 72.402 \\
\hline
\end{tabular}

Source: own calculation

After verifying the suitability of the data and determining the number of factors, the solution of the factor analysis itself follows. The result is a matrix of correlation coefficients between indicators and factors. Values of the matrix are referred to as factor saturations and explain how the factor significantly affects the indicator and, on the other hand, how the factor significantly indicates the factor. The unrotated matrix covers the most significant variability of the original 
variables. To ensure adequate interpretation, it is essential that each indicator has one higher factor saturation value in only one factor. Due to the high factor saturation between the indicators and several factors, it is necessary to rotate the factors. In the SPSS program, select Varimax - orthogonal rotation and, for a better overview, re-enter the order by size. In addition to varimax within orthogonal rotations, it is also possible to choose, for example, quartimax or equamax. There are also oblique rotations of promax and direct oblimin. The choice of rotation depends on the relationship between the factors. In our case, we assume that the factors will be independent, so we chose the orthogonal rotation of varimax, which is among the three most commonly used and most recommended. After summarizing the results and evaluating the individual indicators, we created Table 2, where the number of factors was chosen based on the variance.

Table 2: Initial Eigenvalues

\begin{tabular}{l|lll|l}
\hline Component & Total & Variance \% & Cumulative variance \% & Factor \\
\hline 1 & 19.774 & 27.463 & 27.463 & Innovative Ability \\
2 & 5.188 & 7.206 & 34.669 & Personality \\
3 & 3.06 & 4.25 & 38.919 & Motivation \\
4 & 2.898 & 4.025 & 42.944 & Team Environment \\
5 & 2.556 & 3.55 & 46.495 & Cooperation \\
6 & 2.324 & 3.228 & 49.722 & Sustainable Development \\
7 & 2.225 & 3.09 & 52.812 & Working characteristics \\
8 & 1.804 & 2.506 & 55.318 & Cognitive abilities \\
9 & 1.665 & 2.313 & 57.63 & Benchmarking \\
10 & 1.543 & 2.143 & 59.774 & Organizational Intelligence \\
11 & 1.464 & 2.034 & 61.807 & E-commerce \\
12 & 1.426 & 1.98 & 63.788 & Knowledge Management \\
13 & 1.358 & 1.887 & 65.674 & Intellectual property \\
14 & 1.28 & 1.778 & 67.452 & Business strategy \\
15 & 1.247 & 1.732 & 69.184 & Commercialization \\
16 & 1.218 & 1.692 & 70.875 & 72.402 \\
17 & 1.099 & 1.526 & & Development of new products \\
\hline
\end{tabular}

Source: own calculation

Regression analysis examines the dependence of the change of the dependent variable $\mathrm{Y}$ in the changes of independent variables $X$. The functions must be linear, and since performed factor analysis, we can assume linearity. Analysis of Variance (ANOVA) investigates the dependence of quantitative variables on one or more factors (Kano, Harada, 2000). In our case, it is a one-dimensional multi-factor analysis of variance. In this section, the null hypothesis tested. We use the F test (Significance F) to evaluate this statement. If the "P" value for the Ftest of overall significance evaluation is less than the significance level $(\alpha=0.05)$, the null hypothesis can be rejected and an alternative accepted. Table 3 provides the necessary information to predict the number of $\mathrm{R} \& \mathrm{D}$ costs as well as to determine whether innovation factors contribute statistically significantly to the model (column "Sig."). In the regression analysis examining dependence, we have established the following hypothesis:

H0: There is no statistically significant regression model of R\&D dependence on innovation factors. 
H1: There is a statistically significant regression model of R\&D dependence on innovation factors.

The model in Table 3 is made up of the innovation factors in which a significant regression model of dependence between R\&D and innovation factors was confirmed, i.e., the null hypotheses were rejected for factors: Innovative ability, Benchmarking, Business Strategy and Personality, and we accepted the alternative hypothesis.

Table 3: Model of dependence between $R \& D$ costs and innovation factors

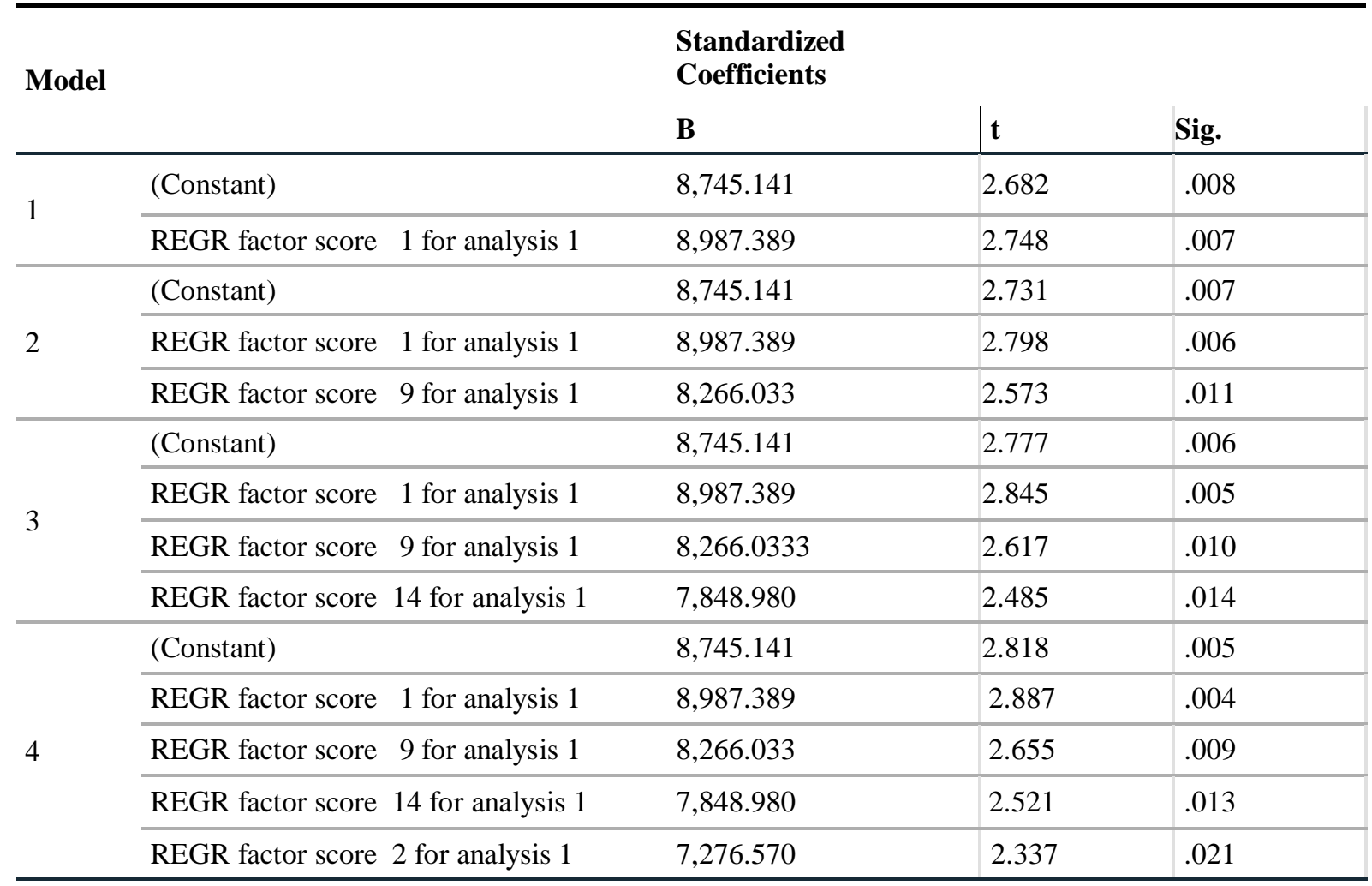

Source: own processing

Based on linear regression, we created a model that is specified in Table 3, by which we can model the relationship between the factor score and the volume of R\&D costs. Explained the dependent variable "Y" represents the amount of research and development costs. Explanatory, in this case, the independent variable " $\mathrm{X}$ " is a factor score. The regression function has the following form:

$$
Y=8,745.141+8,987.389 X 1+8,266.033 X 9+7,848.980 X 14+7,276.570 X 2
$$

This relationship represents a pairwise linear deterministic (each change of variable $\mathrm{x}$ represents a certain change of variable y, and no other deviations occur) mathematical model. Based on non-standardized coefficients, the constant "Y" allows us to predict the impact of the innovation score on R\&D. If the factor of innovative ability (X1) is increased by one unit, this will lead to an increase in R\&D costs of $€ 8,987.389$. Research and development costs will increase by $€ 8,226.003$ if the benchmarking factor score increases by one unit. The business strategy factor score (X14) and its change by one unit will increase R\&D costs by $€ 7,848,980$. If the Personality (X2) Factor Score is increased by one unit, R\&D costs will increase by $€$ 7,276,570. The "ceteris paribus" rule also applies. 


\section{Discussion}

In the factor analysis, we focused primarily on the study of literature to identify possible factors to support the company's innovation. We have analysed over 100 publications from reputable databases based on keywords. Subsequent analysis of publications, we tried to identify the individual factors of innovation, which we categorized in the theoretical part at particular levels (individual, team, and organizational). Subsequently, we performed a factor analysis that reduced 72 variables to 17 innovation factors, which was the main objective. Within each factor, the technique obtained maximum standard deviations from all variables and determined a common factor score. The identified factor score can thus be used in further analyses. To demonstrate the possible use of the factor score, we also dealt with the creation of a model example using linear regression. We have found a significant correlation between the four innovation factors and R\&D costs, and non-standardized coefficients can predict the impact of innovation factors on R\&D costs.

Subsequent analysis of publications, we tried to identify the individual factors of innovation, which we categorized in the theoretical part at particular levels (individual, team, and organizational). Subsequently, we performed a factor analysis that reduced 72 variables to 17 innovation factors, which was the main objective. Within each factor, the technique obtained maximum standard deviations from all variables and determined a common factor score. The identified factor score can thus be used in further analyses. To demonstrate the possible use of the factor score, we also dealt with the creation of a model example using linear regression. We have found a significant correlation between the four innovation factors and R\&D costs, and non-standardized coefficients can predict the impact of innovation factors on R\&D costs.

\section{Conclusion}

Nowadays, managers are facing many pressures related to changing the business environment or customer requirements RoutledgePinho (2008). The changing environment and requirements thus create space for the innovation-oriented enterprises in order to compete with domestic and foreign enterprises Çelikel (2009). Innovation Management is an enterprise-wide initiative that can integrate the different capabilities and resources of a business to meet the needs of existing customers and attract new customers Romijn and Albaladejo (2002). In the context of innovation management, it is vital to know the incentives or factors that, in many cases, provide a competitive advantage and sustainable business growth Abbas and Raja (2015). Therefore, it was necessary to analyse literature and publications first and foremost, focusing on reputable foreign journals.

The factor analysis was preceded by a summary of the adequacy of the data, based on which we verified the suitability of the created database, and subsequently, we continued the analysis. After identifying several key innovation drivers from the original 72 factors, we have obtained 17 key ones that explain almost $73 \%$ of the total variability. We performed a non-rotated component matrix. Still, then we rotated the component matrix to maximize the indicator load on one of the factors since the unsaturated matrix identified the factor saturation for several factors. The last step of the factor analysis was the determination of the factor score, which can be used in other analyses. We handled the factor score results to create a model of the relationship between R\&D costs and key innovation factors. From 17 identified factors, we have identified just four key innovation factors, where significant relationships and model results have been confirmed. It can be used to predict $R \& D$ costs if some of the important innovation factors will change. 


\section{Acknowledgement}

The paper is an output of the science project VEGA 1/0428/17 Creation of new paradigms of financial management at the threshold of the 21 st century in conditions of the Slovak Republic.

\section{References}

Abbas, M. \& Raja, U. (2015). Impact of psychological capital on innovative performance and job stress. Canadian Journal of Administrative Sciences/Revue Canadienne des Sciences de l'Administration, 32(2), 128-138.

Bogue, R. (2016). Growth in e-commerce boosts innovation in the warehouse robot market. Industrial Robot: An International Journal, 43(6), 583-587.

Çelikel, A.T. (2009). Factors affecting research and development (R\&D) collaboration of multinational enterprises (MNEs) and their local partner firms: A case study of Turkish automotive industry.

Coccia, M. (2017). Sources of technological innovation: Radical and incremental innovation problem-driven to support competitive advantage of firms. Technology Analysis \& Strategic Management, 29(9), 1048-1061.

Cooper, R.G. \& Edgett, S.J. (2010). Developing a product innovation and technology strategy for your business. Research-Technology Management, 53(3), 33-40.

Dobni, C.B. (2008). Measuring innovation culture in organizations: The development of a generalized innovation culture construct using exploratory factor analysis. European Journal of Innovation Management, 11(4), 539559.

Foroudi, P., Jin, Z., Gupta, S., Melewar, T.C. \& Foroudi, M.M. (2016). Influence of innovation capability and customer experience on reputation and loyalty. Journal of Business Research, 69(11), 4882-4889.

Garcia-Granero, A., Llopis, O., Fernandez-Mesa, A. \& Alegre, J. (2015). Unraveling the link between managerial risk-taking and innovation: The mediating role of a risk-taking climate. Journal of Business Research, 68(5), 1094-1104.

Goodwyn, F. (2012). Factor Scores, Structure Coefficients, and Communality Coefficients. Online Submission.

Kaasa, A. (2016). Culture as a possible factor of innovation: Evidence from the European union and neighboring countries. Re-thinking Diversity. Management - Culture - Interpretation. Springer VS, Wiesbaden.

Kang, M. \& Lee, M.J. (2017). Absorptive capacity, knowledge sharing, and innovative behaviour of R\&D employees. Technology Analysis \& Strategic Management, 29(2), 219-232.

Kano, Y. \& Harada, A. (2000). Stepwise variable selection in factor analysis. Psychometrika, 65(1), 7-22.

Klami, A., Virtanen, S., Leppäaho, E. \& Kaski, S. (2015). Group factor analysis. IEEE Transactions on Neural Networks and Learning Systems, 26(9), 2136-2147.

Kratzer, J., Meissner, D. \& Roud, V. (2017). Open innovation and company culture: Internal openness makes the difference. Technological Forecasting and Social Change, 119, 128-138.

Neumann, C.S., Kosson, D.S. \& Salekin, R.T. (2017). Exploratory and confirmatory factor analysis of the psychopathy construct: Methodological and conceptual issues. Psychopath, 79-104.

RoutledgePinho, J.C. (2008). TQM and performance in small medium enterprises: The mediating effect of customer orientation and innovation. International Journal of Quality \& Reliability Management, 25(3), 256275.

Romijn, H. \& Albaladejo, M. (2002). Determinants of innovation capability in small electronics and software firms in southeast England. Journal of Research Policy, 31(7), 1053-1067.

Schepers, J.J., Nijssen, E.J. \& van der Heijden, G.A. (2016). Innovation in the frontline: Exploring the relationship between role conflict, ideas for improvement, and employee service performance. International Journal of Research in Marketing, 33(4), 797-817.

Schewe, G. (1991). Key factors of successful innovation management (No. 274). Manuskripte aus den Instituten für Betriebswirtschaftslehre der Universität Kiel.

Terziovski, M. (2010). The relationship between innovation management practices and innovation performance in the mainstream: An empirical study of Australia organisations. In Proceedings of ANZAM Conference 2010. ANZAM.

Thompson, B. (1993). Calculation of standardized, noncentered factor scores: An alternative to conventional factor socres. Perceptual and Motor Skills, 77, 1128-1130.

Walker R.M., Avellaneda C.N. \& Berry F.S. (2011). Exploring the diffusion of innovation among high and low innovative localities: A test of the berry and berry model. Public Management Review, 13(1), 95-125. 